\title{
EDUCACIÓN SANITARIA PARA LA PREVENCIÓN DE ENFERMEDADES: REVISIÓN DE LA LITERATURA
}

\section{ARTÍ́CULO DE REVISIÓN}

GITIRANA, José Valdeci Almeida1, FONSECA, Rosa Maria Batista Pinheiro da², PILONETO, Fábio Marmentini ${ }^{3}$, BEVILAQUA, Luis Felipe Gaia, ASSIS, Ingrid de ${ }^{4}$, CARDOSO, Ronald de Oliveira ${ }^{5}$

GITIRANA, José Valdeci Almeida. Et al. Educación sanitaria para la prevención de enfermedades: revisión de la literatura. Revista Científica Multidisciplinar Núcleo do Conhecimento. Año. 06, Ed. 11, Vol. 08, págs. 134-147. Noviembre de 2021. ISSN: 2448-0959, Enlace de acceso: https://www.nucleodoconhecimento.com.br/salud/educacion-sanitaria, DOI: 10.32749/nucleodoconhecimento.com.br/salud/educacion-sanitaria

\section{RESUMEN}

Actualmente se llevan a cabo políticas públicas, acciones gubernamentales y diferentes programas de Educación sanitaria (ES) a favor de la salud de la población. Con respecto al tema de la ES, se trata de un tema multifacético, convergente a diversas concepciones personales, ya sea en el área de la educación - la salud, que puede dar lugar a entendimientos divergentes. Debido a la complejidad del tema, el problema se produce debido a la falta de comprensión de lo que realmente es la ES orientada a la población, disolviendo la importancia de la ES para las medidas preventivas contra diferentes enfermedades, lo que genera un alto costo presupuestario en la salud pública. En vista de este contexto, este artículo tiene como pregunta principal: ¿Cómo puede la Educación sanitaria contribuir a la

\footnotetext{
${ }^{1}$ Licenciado en Farmacia, postgrado en Análisis Clínicos, Actualmente académico médico.

${ }^{2}$ Un estudioso de la medicina.

${ }^{3}$ Graduado en administración de empresas. Licenciado en Comercio Exterior, actualmente estudiante de medicina.

${ }^{4}$ Un estudioso de la medicina.

${ }^{5}$ Un estudioso de la medicina.
} 
prevención de enfermedades en la población? El estudio tuvo como objetivo presentar el ES para la Prevención de Enfermedades (PD), ramificando en el abordamiento de sus conceptos, así como destacando las bases legales que aseguran el ES a la población. Con este fin, el estudio se realizó a través de una revisión de la literatura por el sitio de búsqueda "Google Scholar", "Scielo" y "PubMed". A través de los descriptores: Educación sanitaria; La Educación sanitaria como prevención de enfermedades; Educación sanitaria de la población, donde se eligieron estudios que presentaron las ES enfocadas en la PD. Se concluye que las ES son la educación para el autoconocimiento y la reflexión sobre su propia salud, así como la plena conciencia de que algo sale mal, atribuyendo la reflexión crítica del sujeto respecto a sus hábitos y medidas preventivas. Se aplica a través del currículo escolar de la escuela primaria, así como por los programas gubernamentales en los centros de salud, con el fin de educar a la sociedad para crear conciencia sobre las medidas preventivas y promover una mejor calidad de vida, evitando así la propagación de enfermedades.

Palabras clave: Educación sanitaria, Prevención de enfermedades, Promoción de la salud, Población.

\section{INTRODUCCIÓN}

La Educación sanitaria (ES) es una práctica social cuyo proceso de aprendizaje contribuye a la formación de una conciencia personal crítica respecto a los propios problemas de salud, haciendo reflexionar al ciudadano sobre su realidad, y a partir de ella, buscar soluciones y organización a favor de su salud y salud colectiva, resultado en una Promoción de la Salud (PS) (BISCARDE; PEREIRA-SANTOS( SILVA, 2014).

Los orígenes y concepciones de PS comenzaron con el advenimiento de ES a principios del siglo 20, a través de la observación de la disminución en las tasas de enfermedad resultante de las prácticas educativas realizadas por los "higienistas" de [7] la época (ENRIA; STAFOLANI, 2010). En ese momento, el significado de PS se atribuyó a las acciones de ES, con el objetivo de una mejor calidad de vida. Sin 
embargo, el ES actualmente tiene una característica amplificada, ya que se considera uno de los principales dispositivos para la viabilidad de PS, lo que lleva al desarrollo de la responsabilidad individual y la prevención de enfermedades (PD) (LOPES; Granizo; XIMENES, 2010; FALKENBERG et al., 2014; SPERANDIO; SPERANDIO, 2018).

Aunque el ES se entiende como una de las medidas para el PD (JANINI; BESSLER; VARGAS, 2015; SPERANDIO; SPERANDIO, 2018), es un tema multifacético, convergente a diversas concepciones personales, ya sea para las personas en el área de la educación, o en el área de la salud, lo que puede resultar en entendimientos divergentes. (SCHALL; STRUCHINER, 1999).

Debido a la complejidad del tema, el problema se produce debido a la falta de comprensión de lo que realmente es la ES orientada a la población, disolviendo la importancia de la ES para las medidas preventivas contra diferentes enfermedades. Debido a esto, muchas enfermedades que podrían evitarse a menudo se evidencian que tienen un gran impacto en el presupuesto público. Por lo tanto, el presente estudio fue para responder a la pregunta: ¿Cómo puede la educación sanitaria contribuir a la prevención de enfermedades en la población? A través de una revisión de la literatura, el objetivo general buscó presentar cómo las ES pueden promover la PD, diversificarse en el abordaje de sus conceptos, así como resaltar las bases legales que aseguran la ES a la población.

Con este fin, el estudio se realizó a través de una revisión de la literatura por el sitio de búsqueda "Google Scholar", "Scielo" y "PubMed". A través de los descriptores: Educación sanitaria; La educación sanitaria como prevención de enfermedades; Educación sanitaria de la población. Como forma de inclusión, se eligieron artículos y documentos entre 2018 y 2020, componiendo la estructura necesaria para lograr el objetivo deseado. Posteriormente, se encontraron 20 artículos, de los cuales se seleccionaron 8. Se incluyeron los artículos que responden al objetivo y a la pregunta guía y que estaban dentro del plazo propuesto. Se excluyeron los estudios dirigidos únicamente a profesionales de la salud o estudiantes de pregrado especializados en el área de la salud. 
El tema es relevante hoy en día, ya que la ES puede influir en la capacidad de reflexión de la población para combatir diferentes enfermedades, lo que resulta en mejores hábitos de vida. Puede ser relevante para los laicos o para los estudiantes y profesionales en el campo académico, sirviendo como fuente para futuros estudios porque está iluminando el concepto de ES para la población como inseparable del $\mathrm{PD}$, proporcionando un lenguaje que es fácil de entender.

\title{
2. LA INDISOCIABILIDAD DE LA EDUCACIÓN SANITARIA Y LA PREVENCIÓN DE ENFERMEDADES
}

La educación superior se planifica y aplica de diferentes formas, dicha modalidad de enseñanza puede estar dirigida a estudiantes de pregrado en el área de la salud; para profesionales de la salud; para la educación escolar y para la población en general. En efecto, tiende a entenderse de diferentes formas, atendiendo a la formación cultural y social de cada uno. Sin embargo, este estudio enfatiza la ES para la población como inseparable de la PD, ya que la ES está "[...] ligada a los objetivos generales de la salud pública: prevención de enfermedades, protección y promoción de la salud del individuo, la familia y la comunidad". (PIMONT, 1997, pág.17).

Para contextualizar mejor la comprensión de esta inseparabilidad, es importante destacar que las ES son una:

\begin{abstract}
Processo educativo de construção de conhecimentos em saúde que visa à apropriação temática pela população [...]. Conjunto de práticas do setor que contribui para aumentar a autonomia das pessoas no seu cuidado e no debate com os profissionais [...] a fim de alcançar uma atenção de saúde de acordo com suas necessidades. (BRASIL, 2012, p. 19).
\end{abstract}

Como se presenta en el "Glosario Temático: gestión del trabajo y Educación sanitaria", el ES causa la autonomía deseable para que las personas puedan expresarse de manera efectiva con el fin de evidenciar sus necesidades específicas en su atención clínica, contribuyendo a la atención del profesional de la salud para tratar rápidamente las enfermedades, así como prevenir otras enfermedades 
secundarias. Complementando esta definición, se presenta en el documento de la Fundación Nacional de Salud (FUNASA) Brasil (2007), que la ES es una práctica social, reafirmando que su intención se teje en una transformación para cualquier tipo de persona "[...] rompiendo con el paradigma de la concepción estática de la educación como transferencia de conocimientos, habilidades y destrezas". (BRASIL, 2007, p. 20).

Pimont (1997, p. 15) resume y analiza los diferentes conceptos adoptados, enfatizando que ES tiene como objetivo proporcionar:

1) A consciência do direito à saúde, dentro do grau de desenvolvimento do grupo social no qual vive o indivíduo; 2) O conhecimento sobre saúde; 3) O conhecimento dos serviços para a saúde que a comunidade possua e sua consequente utilização; 4) O conhecimento e a utilização das práticas de saúde.

Sin embargo, el autor asimila que para que esta enseñanza se transforme en conocimiento con el fin de promover LA y la DP, es necesario que se estructure una planificación, atenuando una metodología educativa adecuada, compuesta por instrumentos auxiliares, que luego puedan resultar en un proceso educativo constante.

Según Altuve (1996), para que la ES se convierta en conocimiento, la organización de la comunidad ya se convierte en un método que produce cambio social, pues en medio de esfuerzos y técnicas, su aplicación resulta en la inducción y promoción de un cambio social significativo, abarcando todos los sectores sociales, como la relación de nivel económico, político y educativo.

Por lo tanto, según los autores citados, la ES puede ser inseparable de la PD a la población, cuando se transmite metodológicamente. Sin embargo, el presente estudio no pretende profundizar en estas metodologías, sino también conceptualizar las ES como PD para la población. 


\subsection{BASES LEGALES PARA LA EDUCACIÓN EN SALUD DE LA POBLACIÓN}

Con un enfoque en es en Brasil, en agosto de 1971 se creó la Ley 5692, que reformula el currículo escolar en la educación primaria y secundaria implementando la educación orientada a la salud como se presenta en el artículo 7:

Art. 7ํㅗㄹ Será obrigatória a inclusão de Educação Moral e Cívica, Educação Física, Educação Artística e Programas de Saúde nos currículos plenos dos estabelecimentos de ํㅡ e $2^{\circ}$ graus, observado quanto à primeira o disposto no Decreto-Lei n. 369, de 12 de setembro de 1969. (Vide Decreto no 69.450, de 1971)

Programas posteriores como "Salud en la escuela", fueron utilizados como una estrategia intersectorial desarrollada en el espacio escolar y su entorno, articulando acciones dirigidas a LA y calidad de vida, junto con la reflexión sobre la pd y el conocimiento del Sistema Único de Salud (SUS) (BRASIL, 2012). Este contexto puede estar marcado por el hecho de que las acciones para las PD se implementan a lo largo de la vida, desde la primera fase de la educación escolar, para que las personas desarrollen un sentido crítico, reflexivo y consciente gradualmente.

Al hacer estallar positivamente el alcance de los programas dirigidos a ES y PD, se crearon diferentes leyes, de acuerdo con las bases legales presentadas en la Tabla 1 a continuación: 
Tabla 1 - Bases jurídicas de la Educación sanitaria.

\begin{abstract}
A Lei n. ${ }^{\circ}$ 8.080/1990, que regulamenta o SUS, em seu Capítulo I, Art. $5^{\circ}$, Item III, define como um dos objetivos e atribuições deste Sistema: "a assistência às pessoas por intermédio de ações de promoção, proteção e recuperação da saúde, com a realização integrada das ações assistenciais e das atividades preventivas". A mesma Lei, em seu Capítulo II, Artigo 7_ ${ }^{\circ}$, Item VIII, estabelece a
\end{abstract} "participação da comunidade."

A Lei $\mathbf{N}^{\circ} \mathbf{8 . 1 4 2}$, de 28 de dezembro de 1990, define a participação da comunidade na gestão SUS e no seu artigo $1^{\circ}$ "as instâncias colegiadas de controle social: I) a Conferência de Saúde, e II) o Conselho de Saúde."

A Norma Operacional Básica - SUS 1/1996 (NOB/96), que redefine as responsabilidades dos estados, do Distrito Federal e da União e consolida as responsabilidades dos municípios, cita como um dos papéis do gestor federal e do estadual a "Educação em Saúde

A Educação em Saúde na Funasa está ancorada em seu Regimento Interno, Portaria GM n ${ }^{\circ} 1.776$ de 8/9/2003, assinada pelo Ministro da Saúde que estabelece as competências da Assessoria de Comunicação e Educação em Saúde-Ascom e seus setores, dentro das competências dos demais setores do órgão. (...)

Lei n. 9.394, de 20 de dezembro de 1996 (1996). Estabelece as diretrizes e bases da educação nacional.

DCN - EF 1988 - Estabelecer as Diretrizes Curriculares Nacionais para o Ensino Fundamental: Saúde articulada à educação e à vida cidadã.

PCN - CN 1988 - Estabelecer as Referências Nacionais Comuns para o Ensino das Ciências Naturais: Saúde como estado de equilíbrio dinâmico do corpo.

PCN - TT Saúde 1988 - Apresentar a saúde como um Tema Transversal: Educação para a saúde

PCN - EM 1999 - Apresentar os Parâmetros Curriculares Nacionais para o Ensino Médio: Degradação ambiental e agravos à saúde humana.

PCN + 2002 - Apresentar Orientações Curriculares complementares aos PCN: Saúde e as condições de vida das pessoas.

OCEM 2006 - Apresentar sugestões para as práticas pedagógicas e organização dos

currículos do Ensino Médio: Ambiente e saúde.

DCNEB 2013 - Estabelecer Orientações Nacionais para a Educação Básica. Saúde como campo de conhecimento e de serviços.

BNCC - 1 ${ }^{a}$ versão 2015 - Estabelecer uma Base Nacional Comum para os currículos da Educação Básica, com versão inicial disponibilizada para consulta pública, estabelecendo cuidado com a saúde da população.

BNCC - 2 ${ }^{\mathrm{a}}$ versão 2016 - Estabelecer uma Base Nacional Comum para os currículos da Educação Básica, com versão elaborada após contribuições da sociedade, para o ambiente e saúde.

BNCC $3^{a}$ versão 2016 - Estabelecer uma Base Nacional Comum para os currículos da Educação Básica com versão disponibilizada após contribuições institucionais, para promoção dos cuidados com a saúde.

BNCC versão final (EI e EF) 2016 - Estabelecer uma Base Nacional Comum para os currículos da Educação Infantil e do Ensino Fundamental, compondo a versão final, com o objetivo de promover os cuidados com a saúde.

BNCC EM 2018 - Estabelecer uma Base Nacional Comum para os currículos do Ensino Médio, promoção do cuidado com a saúde.

Fonte: Adaptado de Brasil (2012); Sousa, Guimarães e Amantes (2019).

RC: 102787

Disponible: https://www.nucleodoconhecimento.com.br/salud/educacion-sanitaria 
El marco presenta las leyes, donde se destacan algunas bases educativas para la aplicabilidad de la ES en la escuela, incluso si tales pautas se centran en el entorno escolar, los propósitos curriculares guían la formación de personas que entienden la importancia de la ES y que a través de este conocimiento pueden promover el cuidado de la salud personal y de la población, también componiendo el benefactor del PD.

Hoy Naciones Unidas presenta el tercer objetivo en la "Agenda 2030", y con apoyo global, se pretende a través de la acción, "Garantizar una vida saludable y promover el bienestar para todos y todas, a todas las edades" (ODS, 2021) independientemente de la clase social, raza o color.

El lugar en la agenda 2030 está en línea con la perspectiva de SHALL (1999), cuando atenúa que la definición de ES debe ser más amplia que simplemente una acción resultante de la PS, ya que se centra en la población pasa por un proceso integral de participación activa de toda la población en el contexto de su vida cotidiana y no solo de las personas que están en riesgo de enfermar, siendo entonces una forma de prevenir enfermedades para las personas sanas, estableciendo una mejor calidad de vida y un mayor bienestar para todos.

\subsection{ESTUDIOS RELACIONADOS CON LA PREVENCIÓN DE ENFERMEDADES A TRAVÉS DE LA EDUCACIÓN SANITARIA}

A continuación, se presentarán los principales hallazgos sobre la DP como resultado de la aplicación de ES, disponibles en la literatura científica, entre los años 2018 y 2020. A continuación, la tabla 2 muestra una síntesis de los 8 artículos previamente seleccionados presentados en orden cronológico, donde se explicarán posteriormente las metodologías utilizadas en cada estudio. 
Tabla 2 - Estudios relacionados con la educación sanitaria como prevención de enfermedades.

\begin{tabular}{|c|c|c|}
\hline Autor & Gol & Conclusión \\
\hline $\begin{array}{l}\text { Dias et al. } \\
(2018)\end{array}$ & $\begin{array}{l}\text { Reportar la experiencia de una } \\
\text { acción de educación sanitaria } \\
\text { con estudiantes de primaria de } \\
\text { una escuela pública en áreas } \\
\text { rurales. }\end{array}$ & $\begin{array}{l}\text { Los niños están expuestos al } \\
\text { riesgo de contaminación por } \\
\text { parásitos, dadas las condiciones } \\
\text { sanitarias que habitan, } \\
\text { evidenciando la necesidad de } \\
\text { ampliar las acciones de educación } \\
\text { sanitaria sobre la importancia de } \\
\text { prevenir los parásitos. }\end{array}$ \\
\hline $\begin{array}{l}\text { Salci, } \\
\text { Meirelles e } \\
\text { Silva (2018) }\end{array}$ & $\begin{array}{l}\text { Comprender cómo se } \\
\text { organizan los miembros de la } \\
\text { Estrategia de Salud de la } \\
\text { Familia para la educación en } \\
\text { salud, en el manejo y } \\
\text { prevención } \\
\text { complicaciones crónicas de la } \\
\text { diabetes mellitus en la Atención } \\
\text { Primaria de Salud }\end{array}$ & $\begin{array}{l}\text { Se considera que la práctica de la } \\
\text { educación sanitaria de las } \\
\text { personas con diabetes } \\
\text { El mellitus en atención primaria de } \\
\text { salud compone un contexto } \\
\text { multifacético, con múltiples } \\
\text { barreras para su ejecución. }\end{array}$ \\
\hline
\end{tabular}

Gomes et Informar sobre la acción El conocimiento sobre el cáncer al. (2019) educativa en un grupo de de pene todavía necesita ser hombres con el fin de buscar mejor trabajado entre los hombres. una mejor comprensión del cáncer de pene y las medidas de prevención y detección temprana.

Figueiredo; Conducir a la creación de Las Políticas Públicas son Antunes e políticas públicas de educación esenciales para la comunicación y De Miranda en salud, con atención escolar el fortalecimiento de todas las 
específica y la debida medidas y lineamientos en las derivación de estudiantes con áreas de educación y salud. La obesidad a unidades de salud interdisciplinariedad promoverá las transformaciones deseadas, a través de acciones de prevención, con el objetivo de reducir la implicación de la obesidad y sus consecuencias, en los grupos de edad más jóvenes, así como la reducción de las enfermedades cardiovasculares en la fase temprana de la vida y en la edad adulta.

Ricci et al. Reportar la experiencia de La realización de la educación (2019) académicos de la Universidad sanitaria sobre las infecciones de Federal de Mato Grosso do Sul (UFMS) durante una acción transmisión sexual (ITS) durante el desarrollada con el grupo de embarazo demostró ser eficaz mujeres embarazadas de una para proporcionar diálogo, Unidad Básica de Salud en interacción, intercambio de Campo Grande - MS (UBS). experiencias, conocimientos y experiencias, y la construcción conjunta de conocimientos, siendo luego evidenciados como importantes para la promoción de la salud y la prevención de enfermedades, y para el desarrollo de una conciencia crítica y reflexiva de las mujeres embarazadas sobre su proceso de salud-enfermedad. haciéndolos activos en el proceso de 


\begin{tabular}{|c|c|c|}
\hline & & aprendizaje. \\
\hline $\begin{array}{l}\text { De Sousa } \\
\text { Gonçalves } \\
\text { et al. (2020) }\end{array}$ & $\begin{array}{l}\text { Conocer la labor de educación } \\
\text { sanitaria de una unidad básica } \\
\text { de salud en el municipio de } \\
\text { Imperatriz - MA. }\end{array}$ & $\begin{array}{l}\text { Se percibe que es fundamental } \\
\text { mejorar constantemente el } \\
\text { conocimiento y la práctica de los } \\
\text { profesionales para desarrollar } \\
\text { acciones de educación sanitaria. } \\
\text { Es necesario ampliar las acciones } \\
\text { educativas dentro del área de } \\
\text { responsabilidad de cada equipo, } \\
\text { así como una participación más } \\
\text { activa de todos los miembros del } \\
\text { equipo de estrategia familiar, así } \\
\text { como de los profesionales del } \\
\text { centro de apoyo a la salud de la } \\
\text { familia (NASF). }\end{array}$ \\
\hline $\begin{array}{l}\text { Lima et al. } \\
(2020)\end{array}$ & $\begin{array}{l}\text { Describir la experiencia como } \\
\text { estudiantes de enfermería en } \\
\text { actividades de educación } \\
\text { sanitaria para la prevención de } \\
\text { enfermedades cardiovasculares } \\
\text { en ancianos. }\end{array}$ & $\begin{array}{l}\text { Las prácticas de educación } \\
\text { sanitaria son transformadoras y } \\
\text { aportan importantes aportes a la } \\
\text { enfermería gerontológica, ya que } \\
\text { posibilita el ejercicio del } \\
\text { pensamiento crítico-reflexivo y } \\
\text { contribuye a la formación de } \\
\text { profesionales comprometidos con } \\
\text { el trabajo en un modelo } \\
\text { asistencial, basado en la evidencia } \\
\text { científica y capaz de satisfacer las } \\
\text { necesidades de la población de } \\
\text { edad avanzada, que ha ido } \\
\text { creciendo a lo largo de los años. }\end{array}$ \\
\hline $\begin{array}{l}\text { Costa et al. } \\
(2020)\end{array}$ & $\begin{array}{l}\text { Informar de una acción de } \\
\text { educación sanitaria ocurrida en }\end{array}$ & $\begin{array}{l}\text { La acción desarrollada sirvió como } \\
\text { mecanismo consolidador de los }\end{array}$ \\
\hline
\end{tabular}




\begin{tabular}{|c|c|}
\hline $\begin{array}{l}\text { el ámbito de la atención } \\
\text { primaria de salud, cuya } \\
\text { propuesta era prevenir el } \\
\text { cáncer de mama desde el } \\
\text { reconocimiento previo de sus } \\
\text { manifestaciones, así como el } \\
\text { mantenimiento de hábitos } \\
\text { saludables, con el objetivo de } \\
\text { promover la salud de estas } \\
\text { mujeres. }\end{array}$ & $\begin{array}{l}\text { aspectos que constituyen la } \\
\text { educación sanitaria, reiterando el } \\
\text { papel fundamental de la } \\
\text { enfermería en el proceso } \\
\text { asistencial. }\end{array}$ \\
\hline
\end{tabular}

Fonte: $O$ autor.

Dias et al. (2018), a través de un informe de experiencia con un método de actividad extraclase del componente curricular "Proyecto Integrador Científico de enfermería de pregrado" de la Facultad Verde Norte, realizado en octubre de 2016 en la Escuela Municipal Odilon Antunes, en la Aldea Poções de la ciudad de Monte Azul, una acción de educación sanitaria con la participación de 30 estudiantes, 03 profesores y 09 padres. La acción se produjo en las etapas de exposición dialógica del contenido y en la visualización del disparador de video discusión, siendo debatida en rueda de conversaciones con la audiencia. Por lo tanto, el estudio proporcionó medidas preventivas para la contaminación por enfermedades parasitarias.

Salci; Meirelles e Silva (2018) realizó un estudio cualitativo, componiendo el Pensamiento Complejo como referencia teórica y la investigación evaluativa como referencia metodológica. Para la recolección de datos, 38 profesionales de la salud entrevistaron a pacientes con Diabetes Mellitus. Los agentes de salud observaron y analizaron las historia clínicas de los pacientes, a través de la triangulación de los datos con el apoyo del software ATLAS.ti. Los autores observaron que de acuerdo con la complejidad del tema multifacético, ES puede promover la PD primaria y secundaria. 
Corroborando esta complejidad, Figueiredo; Antunes y De Miranda (2019) realizaron una investigación de revisión bibliográfica, aplicando el método de investigación exploratoria. Los autores relacionaron la prevención de comorbilidades y enfermedades cardiovasculares con las políticas públicas de ES en el ámbito escolar. Por ello, destacaron que la interdisciplinariedad que implica la ES puede promover las transformaciones deseadas en los estudiantes, a través de acciones de prevención, previniendo enfermedades cardiovasculares en la infancia y la edad adulta.

Lima et al. (2020) en un estudio similar, pero dirigido al público anciano, observaron a través de un informe de experiencia, a través del método descriptivo, con un enfoque cualitativo, experimentado en el "Programa de Atención de Salud para Ancianos - PROASI", que las actividades de ES sirven como una herramienta en la prevención de enfermedades cardiovasculares, causando un pensamiento críticoreflexivo a su propia salud y calidad de vida.

Gomes et al. (2019) a través del estudio descriptivo exploratorio del tipo de relato de experiencia, aplicaron una actividad realizada en marzo de 2018 en una iglesia ubicada en las afueras de Belém/Pará. El público participante estuvo compuesto por veintitrés hombres de entre 18 y 50 años que fueron sometidos a conferencias con la presentación de pancartas; explicación del cáncer de pene; cuidado de la PD y demostración de prótesis de pene. Los autores observaron a través de la rueda de conversación con los participantes, que pocos conocían sobre el tema del cáncer de pene. Por ello, los autores advirtieron sobre la importancia de implementar programas de ES enfocados en la PD como el cáncer de pene, pues con programas enfocados en ES esta y otras enfermedades pueden disminuir considerablemente en la población.

Todavía en el contexto de la prevención del cáncer, Costa et al. (2020), realizó un estudio descriptivo con enfoque cualitativo, del tipo de informe de experiencia. El estudio se realizó en el ámbito hospitalario, encontrado en la región periférica de la ciudad de Belém-PA, en una unidad de salud con profesionales que promueven una modalidad de tratamiento de salud enfocada en el nivel primario de enfermedades. 
Los tratamientos dirigidos al público femenino se consolidan en la implementación del "Programa Saúde da Mulher", en el que las pacientes son tratadas con atención prenatal; parto; posparto; cuidado de las familias; programas para la prevención del cáncer de mama y de útero; IST. Este programa está compuesto por profesionales de diversas áreas de la salud, junto con los diversos tipos de análisis clínicos para la adopción de medidas preventivas, promoviendo el diagnóstico previo de patologías como el cáncer. Con la implementación de una acción educativa en el programa, los profesionales de la salud siguieron a los pacientes en sus consultas, y de manera similar al estudio de Gomes et al. (2019) carpetas expuestas; presentaciones anatómicas de la mama; entre otras actividades educativas e interactivas. Por ello, los autores aclararon que las 16 participantes tuvieron una mayor reflexión sobre la importancia del autocuidado y comprendieron la importancia de la educación superior para la prevención del cáncer de mama.

Con respecto a esta reflexión citada por Gomes et al. (2019), Ricci et al. (2019) corroboran que tal proceso de conciencia reflexiva se produce a través del diálogo; interacción; intercambio de experiencias; conocimientos y experiencias y la construcción conjunta del conocimiento, siendo entonces, tales elementos evidenciados como importantes para el PS y el PD. Esta observación se evidenció a través del estudio ocurrido el 15 de marzo de 2017, en la primera noche en la sala de reuniones de una Unidad Básica de Salud de Campo Grande - MS. Se utilizó una metodología de manera estratégica compuesta por prácticas de ludicidad con el objetivo de interactividad de los participantes para que a través de la práctica pedagógica pudieran adquirir la comprensión del tema en cuestión. Inicialmente, las participantes embarazadas fueron invitadas a través de convocatorias de agentes comunitarios de la región de la unidad de salud. Posteriormente se inició la acción con la técnica rompehielos "Pasando al bebé", en la que se presentaba a los participantes identificándose con su nombre, edad gestacional y elección del nombre del niño (a). A través de la dinámica, las mujeres embarazadas tomaron conciencia sobre las enfermedades que podrían prevenirse durante el embarazo, así como la PD en el propio bebé. 
Para que esta conciencia reflexiva involucre a la familia, De Sousa Gonçalves et al. (2020), llevó a cabo un trabajo cuantitativo con enfoque analítico, basado en una recopilación retrospectiva de las acciones de ES realizada de febrero a septiembre de 2019. La investigación se llevó a cabo en una Unidad Básica de Salud (UBS) del municipio de Imperatriz en el estado de Maranhão, en la que estuvo compuesta por 3 equipos de estrategia de salud familiar. Los datos fueron recolectados de la recolección de documentos con registros de las acciones de las ES realizadas en la UBS y en las escuelas del área, cubiertas por cada equipo en el período antes mencionado, puestos a disposición por la dirección de la unidad de salud. Los autores analizaron que a través de la ES no solo se pueden prevenir enfermedades crónicas, sexuales o infecciosas, sino que también pueden resultar medidas profilácticas de programas que ayuden a estas familias.

\section{CONSIDERACIONES FINALES}

ES puede promover la PD a través de programas de políticas públicas destinados a implementar programas educativos de ES en escuelas, UBS, redes hospitalarias y puestos de salud. Para ello, sus conceptos pueden ser ampliamente presentados, ya que ES es un tema complejo y multifacético.

A través de los hallazgos, se considera que la ES puede estimular la PD junto con la PS para la población, cuando existe la participación de este público objetivo a través de actividades metodológicamente planificadas y aplicadas con la contribución de instrumentos específicos para el desarrollo del aprendizaje. En cuanto a la PD, hay varias profilaxis encontradas en los estudios: cardiovasculares, diabetes, enfermedades crónicas no transmisibles, enfermedades infecciosas de transmisión sexual, enfermedades parasitarias, cánceres, entre otros.

En el contexto de las leyes y bases legales para la ES, está dirigido a una gran expectativa en los estudiantes de las etapas tempranas y secundarias, ya que el proceso educativo no se impone en la edad adulta sino que se puede construir durante el curso de la vida, convirtiéndose en conocimiento. 
Por lo tanto, en respuesta a la pregunta del pensamiento correcto, se concluye que los ES son la educación para el autoconocimiento y la reflexión sobre su propia salud, así como la plena conciencia de que algo sale mal, atribuyendo la reflexión crítica del sujeto con respecto a sus hábitos y medidas preventivas, previniendo así las enfermedades. Se aplica a través del currículo escolar de la escuela primaria, así como por los programas gubernamentales en los centros de salud, con el fin de educar a la sociedad para crear conciencia sobre las medidas preventivas y promover una mejor calidad de vida.

\section{REFERENCIAS}

ABREU JUNIOR, Laerthe de Moraes; CARVALHO, Eliane Vianey de. O discurso médico-higienista no Brasil do início do século XX. Trabalho, Educação e Saúde, v. 10, p. 427-451, 2012.

ALTUVE. R. L. O. La organización de la comunidad como un proceso educativo. Cuadernos de la Escuela de Salud Pública (Caracas) 4:9-28, 1966

BISCARDE, Daniela Gomes dos Santos; PEREIRA-SANTOS, Marcos; SILVA, Lília Bittencourt. Formação em saúde, extensão universitária e Sistema Único de Saúde (SUS): conexões necessárias entre conhecimento e intervenção centradas na realidade e repercussões no processo formativo. Interface-Comunicação, Saúde, Educação, v. 18, p. 177-186, 2014.

BRASIL. Fundação Nacional de Saúde. Diretrizes de educação em saúde visando à promoção da saúde: documento base - documento I/Fundação Nacional de Saúde -Brasília: Funasa, 2007.

. Ministério da Saúde (MS) Glossário temático: gestão do trabalho e da educação na saúde. Brasília DF, 2012. Disponível em: https://bvsms.saude.gov.br/bvs/publicacoes/glossario_gestao_trabalho_2ed.pdf Acesso em: 03/07/2021. 
. Lei; LEI, №. 5.692, DE 11 DE AGOSTO DE 1971. Fixa Diretrizes e Bases para o ensino de, v. 1, p. 6377, 1971.

- Lei n. 9.394, de 20 de dezembro de 1996 (1996). Estabelece as diretrizes e bases da educação nacional. Brasília, DF: Ministério da Educação. Disponível em: http://portal.mec.gov.br/seed/arquivos/pdf/tvescola/leis/ lein9394.pdf Acesso em 20 de ago. 2021.

COSTA, Paula Valéria Dias Pena et al. A educação em saúde como ferramenta no combate ao câncer de mama: relato de experiência. Research, Society and Development, v. 9, n. 10, p. e6389108912-e6389108912, 2020.

DE SOUSA GONÇALVES, Romário et al. Educação em saúde como estratégia de prevenção e promoção da saúde de uma unidade básica de saúde. Brazilian Journal of Health Review, v. 3, n. 3, p. 5811-5817, 2020.

DIAS, Ernandes Gonçalves et al. Promoção de saúde na perspectiva da prevenção de doenças parasitárias por meio da educação em saúde com escolares do ensino fundamental. Revista de Epidemiologia e Controle de Infecção, v. 8, n. 3, p. 283285, 2018.

ENRIA, G.; STAFFOLANI, C. Contradicciones de los discursos que dificultan la transformación de las prácticas de promoción a la salud. Hacia la promoción de la salud, Calle, v. 15, n. 1, 2010.

FALKENBERG, Mirian Benites et al. Educação em saúde e educação na saúde: conceitos e implicações para a saúde coletiva. Ciência \& Saúde Coletiva, v. 19, p. 847-852, 2014.

FIGUEIREDO, Elisabeth Almeida; ANTUNES, Daniella Cavalcante; DE MIRANDA, Maria Geralda. Políticas públicas de educação em saúde para a prevenção de comorbidades e doenças cardiovasculares. Revista da Seção Judiciária do Rio de Janeiro, [S.I.], v. 23, n. 45, p. 141-160, jul. 2019. ISSN 2177-8337. 
GOMES, Ana Cláudia Ferreira et al. Educação em saúde para prevenção do câncer de pênis: relato de experiência. Brazilian Journal of Health Review, v. 2, n. 4, p. 2961-2964, 2019.

JANINI, Janaina Pinto; BESSLER, Danielle; VARGAS, Alessandra Barreto de. Educação em saúde e promoção da saúde: impacto na qualidade de vida do idoso. Saúde em Debate, v. 39, p. 480-490, 2015. LOPES, M. S. V.; SARAIVA, K. R. O.;

LIMA, D. C.; GARCIA, M. P. .; LIMA, E. S. de .; BEZERRA, C. C. . Health education as a tool for the prevention of cardiovascular diseases in the Elderly Health Care Program. Research, Society and Development, [S. I.], v. 9, n. 10, p. e079107382, 2020. DOI: 10.33448/rsd-v9i10.7382. Disponível em: https://rsdjournal.org/index.php/rsd/article/view/7382. Acesso em: 28 aug. 2021.

OBJETIVOS DA ONU - ODS. Objetivo 3. Assegurar uma vida saudável e promover o bem-estar para todas e todos, em todas as idades. Disponível em: <https://gtagenda2030.org.br/ods/ods3/>. Acesso em: 25 ago. 2021

PIMONT, Rosa Pavone. A educação em saúde: conceitos, definições e objetivos. Boletín de la Oficina Sanitaria Panamericana (OSP); 82 (1), ene. 1977, 1977.

RICCI, Ana Patrícia et al. Infecções sexualmente transmissíveis na gestação: educação em saúde como estratégia de prevenção na atenção básica. Brazilian Journal of Health Review, v. 2, n. 1, p. 565-570, 2019.

SALCI, Maria Aparecida; MEIRELLES, Betina Hörner Schlindwein; SILVA, Denise Maria Guerreiro Vieira da. Educação em saúde para prevenção das complicações crônicas do diabetes mellitus na atenção primáriaa. Escola Anna Nery, v. 22, 2018.

SCHALL, Virgínia T.; STRUCHINER, Miriam. Educação em saúde: novas perspectivas. 1999.

SOUSA, Marta Caires; GUIMARÃES, Ana Paula Miranda; AMANTES, Amanda. A saúde nos documentos curriculares oficiais para o ensino de ciências: da lei de 
diretrizes e bases da educação à base nacional comum curricular. Revista Brasileira de Pesquisa em Educação em Ciências, p. 129-153, 2019.

SPERANDIO, Naiara; SPERANDIO, Natália Elvira. A educação em saúde enquanto prática transformadora da realidade social. Educação e Saúde: fundamentos e desafios, v. 1, n. 2, p. 7-14, 2018.

XIMENES, L. B. Análise do conceito de promoção da saúde. Texto Contexto Enferm., Florianópolis, v. 19, n. 3, jul./set. 2010, p. 461-468.

\section{APÉNDICE - NOTA DE REFERENCIA}

7. Personas que formaban parte de la doctrina del higienismo, doctrina nacida a mediados del siglo XIX, cuando los gobernantes prestaban mayor atención a la salud, junto con la moral de la población. En ese momento la enfermedad era considerada un fenómeno social que involucra todos los aspectos de la vida humana, es a través de este contexto que surge el tratamiento del agua, alcantarillado, alumbrado público, con el fin de controlar o eliminar las pandemías (ABREU JUNIOR; CARVALHO, 2012).

Enviado: Septiembre de 2021.

Aprobado: Noviembre de 2021. 